\title{
Relation Between Relative Handgrip Strength, Chronological Age and Physiological Age with Lower Functional Capacity in Older Women
}

This article was published in the following Dove Press journal: Open Access Journal of Sports Medicine

\author{
Wylker Souza Saraiva' \\ Jonato Prestes (D) \\ Silvana Schwerz Funghetto $\mathbb{D}^{2}$ \\ James Wilfred Navalta $\mathbb{D}^{3}$ \\ Ramires Alsamir Tibana $\mathbb{D}^{\prime}$ \\ Dahan da Cunha \\ Nascimento (D) ${ }^{1,4}$ \\ 'Department of Physical Education, \\ Catholic University of Brasilia (UCB), \\ Brasilia, Brazil; ' 2 Department of Nursing, \\ University of Brasilia (UNB), Brasilia, \\ Brazil; ${ }^{3}$ Department of Kinesiology and \\ Nutrition Sciences, University of Nevada, \\ Las Vegas, NV, USA; ${ }^{4}$ Department of \\ Physical Education, Center University of \\ Distrito Federal (UDF), Brasilia, Brazil
}

Correspondence: Dahan da Cunha Nascimento

Programa de Pós-Graduação Stricto Sensu em Educação Física, Universidade Católica de Brasília, Q.S. 07, Lote 0I, EPTC - Bloco G, Distrito Federal, Brasilia 7|966-700, Brazil

$\mathrm{Tel} / \mathrm{Fax}+2 \mathrm{I} / 55 / 6133569350$

Email dahanc@hotmail.com
Purpose: Relative handgrip strength (RHGS), Aged Based on Exercise Stress Testing (A-BEST), and chronological age were evaluated as predictors of impaired mobility in older women.

Methods: Participants included 88 older women (mean age $68.13 \pm 6.02$ years) referred for exercise stress testing. Estimated physiological age was computed based on exercise capacity, chronotropic reserve index, heart rate recovery, and medication that could affect heart rate. RHGS was measured using a validated handgrip hydraulic dynamometer and mobility was evaluated by timed up and go test (TUG-test). A hierarchical multiple regression predicted TUG-test performance from A-BEST, chronological age and RHGS.

Results: After adjustment for diabetes, RHGS was the only variable to add significantly to the prediction model $(\mathrm{p}=0.001)$. An increase in RHGS of $1 \mathrm{~kg} /$ body mass index was associated with a decrease in TUG-test of 0.7 seconds.

Conclusion: Relative handgrip strength test was a better predictor of impaired mobility when compared with chronological and physiological age in older women. Moreover, RHGS represents an inexpensive, simple, portable, noninvasive measurement for a clinician when compared with an exercise stress testing.

Keywords: muscle strength, biological age, chronological age, older, functional capacity

\section{Introduction}

The aging process is associated with progressive decline in muscle strength, power, impaired balance, altered cardiac function and vascular function. ${ }^{1}$ These alterations negatively affect exercise capacity, increase cardiovascular disease risk, and have implications for physical function and risk of falling. ${ }^{1}$ Furthermore, an increase in the population aged 60 years and over is projected to increase from $13.8 \%$ in 2020 to $29.4 \%$ in 2050 according to data from the Brazilian Institute of Geography and Statistics. $^{2}$

Chronological age is considered to be a good predictor of health status, while considerable inter-individual variability has been reported, with some older people displaying very good health, and others showing the accelerated onset of weakness, disability and frailty. ${ }^{3}$ Compounding this burden, given that aging is the progressive decline of the organism, the rate is not universal, and as a result, age when measured chronologically might not be a reliable indicator of the progressive decline of body's function. ${ }^{4}$ For example, age is not different between subjects with higher and lower grip strength at baseline, and a weak grip strength has 
a significant negative relationship with future physical status, as lower $3 \mathrm{~m}$ time up and go test (TUG-test). ${ }^{5}$

Thus, different methods were developed to provide a better predictive of health status than chronological age. ${ }^{4}$ Clearly, there is a need to objectively and quantitatively evaluate the importance of each biomarker of aging that is directly correlated with the body's rate of decline breakdown. ${ }^{6}$ Considering that, every biological process might change with age as, muscle strength, mobility (slower walking), and muscle mass. ${ }^{3}$ Thus, every biological parameter can be considered a "biomarker of aging". 6

Physiological age, also known as, the combination of a number of varying biomarkers as, C-reactive protein, serum creatinine and systolic blood pressure, can be a more reliable predictor of mortality and later life depression than chronological age and might facilitate preventative interventions for health. ${ }^{4,7}$ However, handgrip strength and gait speed (a measure of mobility and risk of falls) should also be measured in older adults, as they provide additional prognostic information regarding cardiovascular mortality and inflammation in addition to traditional risk factors included in a number of varying mathematical algorithms used to estimate the physiological age. ${ }^{4,8,9}$ Furthermore, older subjects with poor mobility and increased risk of falls, generally have a poorer state of health (e.g. low muscle strength and mobility) and are more heavily medicated.

The timed up and go test (TUG-test) is an objective, inexpensive, quick and easy method to perform and assess mobility, and also to predict the risk of falls in communitydwelling older adults. ${ }^{10,11}$ Intrinsic factors that cause falls in older subjects include; age, vertigo, lower extremity weakness, diabetes, antidepressants, syncope and stroke, but most of the intrinsic factors previously cited were not used for the estimate of physiological age and a significantly greater number of fractures are the result from falls caused by an intrinsic cause. ${ }^{12}$ Thus, it is important to bear in mind that the role of health professionals is to identify inexpensive and simple biomarkers of age associated with reduced mobility and falls in older subjects.

Moreover, the measure of handgrip strength does not require highly trained personal, is an inexpensive tool that is a simple, portable, noninvasive measurement, and seems relevant in the screening of older subjects with poor health outcomes (e.g. hospitalization, disability, fracture, stroke and all-cause mortality). ${ }^{8,13-15}$

Nonetheless, studies use different risk factors to estimate the physiological age (i.e. cardiovascular and immune). Recently, peak estimated metabolic equivalent of a task, abnormal heart rate recovery (AHRR), chronotropic reserve index (CRI), and medications that affect heart rate (beta-blocker and calcium channel antagonists) were used to estimate patients' physiological age based on exercise stress testing performance and the Aged Based on Exercise Stress Testing (A-BEST), or physiological age, was the best predictor of mortality when compared with chronological age. ${ }^{16}$ However, most older individuals are unable to satisfactorily complete a treadmill exercise test. $^{17}$

While stress tests are not applicable for the vast majority of older persons who are interested in enhancing their physical function through a program of physical activity, ${ }^{17}$ evaluation of handgrip strength might provide additional prognostic information regarding poor health outcomes (i.e. mobility), all-cause mortality and cardiovascular mortality in older adults of multiple nationalities and ethnicities in addition to traditional risk factors included in a number of varying mathematical algorithms used to estimate the physiological age. ${ }^{8}$

As a weak handgrip strength is associated with lower physical performance (i.e. 10-m gait time and TUG-test), impaired heart rate recovery and low chronotropic index. ${ }^{5,18}$ Also, is a stronger predictor of death than systolic blood pressure, even after adjustments for age, sex, country income level, education level, employment status, tobacco, alcohol use, diabetes, heart failure, coronary artery disease, chronic obstructive, pulmonary disease, self-reported prior stroke, self-reported prior cancer, body mass index, and waist-to-hip ratio. ${ }^{14}$

In this evaluation, additional clinical inexpensive tools that are simple, portable and noninvasive measurements as handgrip strength test are needed to create clinically applicable information for the evaluation of reduced mobility in older subjects. Thus, we sought to compare the efficiency of handgrip strength with chronological age and A-BEST in estimating declines in mobility assessed by the TUG-test in older women. The initial hypothesis is that higher handgrip strength better predicts impaired mobility when compared with chronological and physiological age in older women.

\section{Methods}

\section{Subjects}

A total of 157 obese older women from a community located in the Federal District, Brazil were assessed for eligibility. To be eligible for participation in this study, 
women needed to be aged 60-100 years with body fat percentages $\geq 30 \%$ as assessed by dual-energy $\mathrm{x}$-ray absorptiometry (DEXA). Of those, 69 were excluded (did not meet inclusion criteria for body fat percentage) leaving 88 participants who met the inclusion criteria. These women were not specifically representative of the Brazilian population, and were recruited on a voluntary basis through posters and lectures about the study. Subjects were interviewed and responded to a medical history questionnaire (past medical history, cardiac risk factors, prior cardiac events and procedures, and osteoarticular disorders), underwent anthropometric measures, answered a questionnaire about lifestyle information, and use of medications. Subjects were classified as hypertensive by diagnostic criteria used in previous studies and diabetes was defined as documented prescription of insulin or other hypoglycemic medications. ${ }^{19-21}$ Characteristics of the study subjects are presented in Table 1.

The present study was approved by the Institutional Research Ethics Committee of the Catholic University of Brasília (UCB) (protocol 45648115.8.0000.5650/2016). The study design and procedures were in accordance with ethical standards and the Declaration of Helsinki. Each subject was fully informed about the risks associated with study participation and gave their written informed consent.

\section{Evaluation of Mobility}

The timed-up and go test consisted of rising from a chair and walking as fast as possible to a cone $3 \mathrm{~m}$ away, circling around the cone, and returning to sit on the chair. ${ }^{10,11}$ Subjects were allowed three trials to perform each test with $1 \mathrm{~min}$ of interval between trials, and received instructions to perform each test as fast as possible without running. Participants initiated the test with their back against the chair and their hands on their hips.

\section{Handgrip Strength}

Handgrip strength was determined by the use of a handgrip Hydraulic dynamometer (Saehan Corp ${ }^{\circledR}$, SH5001, S. Korea). Three measures on the right and left hand were obtained and the highest value was recorded. The second position was used for all the subjects; with the forearm in a neutral position, elbow fully extended; standing position; and verbal encouragement was used for all subjects with one-minute rest intervals between measurements. To calculate the relative handgrip strength (RHGS), the highest reading from each hand was divided by the
Table I Subjects' Characteristics

\begin{tabular}{|l|l|}
\hline Subject Variables & Overall $(\mathbf{n}=\mathbf{8 8})$ \\
\hline Clinical & \\
Age, mean \pm SD, years & $68.13 \pm 6.02$ \\
Height, mean \pm SD, $\mathrm{m}$ & $1.54 \pm 6.1 \mathrm{I}$ \\
Body weight, mean \pm SD, $\mathrm{kg}$ & $68.55 \pm 11.32$ \\
Body mass index, mean $\pm \mathrm{SD}, \mathrm{kg} / \mathrm{m}^{2}$ & $28.83 \pm 4.39$ \\
Body fat, \% & $39.82 \pm 6.16$ \\
RHGS, mean \pm SD, $\mathrm{m}^{2}$ & $1.70 \pm 0.48$ \\
Absolute HGS, mean $\pm \mathrm{SD}, \mathrm{kg}$ & $24.61 \pm 4.49$ \\
Timed up and go, mean $\pm \mathrm{SD}$, seconds & $6.85 \pm 0.85$ \\
\hline Medications* & \\
Angiotensin receptor blockers & $36(40.91)$ \\
Diuretics & $38(43.18)$ \\
$\beta$-blockers & $15(17.05)$ \\
Calcium channel antagonists & $9(10.23)$ \\
Angiotensin-converting enzyme inhibitors & $16(18.18)$ \\
Statins & $24(27.27)$ \\
Hypoglycemic Medications & $16(18.18)$ \\
\hline Disease* & \\
Hypertension & $64(72.73)$ \\
Diabetes mellitus type 2 & $16(18.18)$ \\
\hline Exercise data & \\
A-BEST, mean \pm SD & $57.23 \pm 2.09$ \\
Resting SBP, mean $\pm \mathrm{SD}, \mathrm{mmHg}$ & $126.31 \pm 15.17$ \\
Resting DBP, mean $\pm \mathrm{SD}, \mathrm{mmHg}$ & $72.71 \pm 8.79$ \\
Peak HR, mean \pm SD, mmHg & $142.72 \pm 16.87$ \\
Peak METs, mean $\pm \mathrm{SD}$ & $5.84 \pm 0.78$ \\
AHRR, $\mathrm{n}$ & 2 \\
CRI, mean \pm SD & $0.80 \pm 0.18$ \\
\hline
\end{tabular}

Note: *Data presented as frequency and percentage values.

Abbreviations: BMI, body mass index; RHGS, relative handgrip strength; MET, metabolic equivalent; SBP, systolic blood pressure; DBP, diastolic blood pressure; $n$, number.

subject's body mass index (BMI). Previous research supports strength corrected for BMI over the absolute strength measures. $^{22,23}$

\section{A-BEST}

To estimate A-BEST we included the same parameters reported in a previous study. ${ }^{16}$ Thus, peak estimated metabolic equivalent of task (METs), abnormal heart rate recovery (AHRR), chronotropic reserve index (CRI), and medications (beta-blocker and calcium channel antagonist) from our subjects were used.

\section{Treadmill Stress Testing}

Exercise testing procedures in the laboratory have been described in detail elsewhere from our research group. ${ }^{24,25}$ 
Subjects underwent a symptom-limited treadmill test using a ramp-treadmill protocol. The protocol used velocity increments (between 0.004 and $0.005 \mathrm{~km} / \mathrm{h}$ each second) and grade (between $0.015 \%$ and $0.021 \%$ each second), adjusted for subjects to reach maximal exercise capacity within the recommended range of $8-12$ mins. The initial and final velocity was $3.0 \mathrm{~km} / \mathrm{h}$ and $6.0 \mathrm{~km} / \mathrm{h}$, respectively, while the initial and final grade was $1.0 \%$ and $14.0 \%$. Subjects were encouraged to exercise until voluntary-exhaustion, and the achievement of $85 \%$ of maximum predicted HR and/or respiratory exchange ratio $>1.02$ was used for the termination of testing. ${ }^{24,25}$ During each exercise stage and recovery stage, symptoms (chest discomfort, rate of perceived exertion, and dizziness), blood pressure, and heart rate were recorded. Following peak exercise (maximum time spent in the test), subjects walked for a 2-min cool-down period at $2.0 \mathrm{~km} / \mathrm{h}$ and $2.5 \%$ grade. $^{26}$ Heart rate recovery was measured during the first and second minutes of the cooldown period and was defined as the difference between heart rate at peak exercise and first minute and second minutes following exercise. Subjects were permitted to lean on the handrails during exercise.

\section{Statistical Analyses}

All statistical analyses were conducted using SPSS software version 18.0 (SPSS Inc., Chicago). A hierarchical multiple regression was utilized to predict TUG-test performance from A-BEST, chronological age and RHGS. The covariate diabetes was also included, as is an independent risk factor for reduced mobility and falls. ${ }^{12}$ There was linearity as assessed by partial regression plots and a plot of studentized residuals against the predicted values. There was independence of residuals, as assessed by a Durbin-Watson statistic of 2.01. There was homoscedasticity, as assessed by visual inspection of a plot of studentized residuals versus unstandardized predicted values. There was no evidence of multicollinearity, as assessed by tolerance values greater than 0.1 . The assumption of normality was met, as assessed by a Q-Q Plot. An alpha level of $\mathrm{p} \leq 0.05$ was considered significant.

\section{Results}

Regression coefficients and standard errors can be found in Table 2 (below).

The multiple regression model statistically significantly predicted TUG-test, $F(4,85)=8.05, p<0.0001$, adj. $R^{2}=$ 0.24 . Only RHGS added significantly to the prediction
Table 2 Summary of Multiple Regression

\begin{tabular}{|l|l|l|l|l|}
\hline \multicolumn{5}{|l|}{ Model } \\
\hline Variable & B & $S_{B}$ & $\boldsymbol{\beta}$ & $\boldsymbol{P}$ \\
\hline Intercept & 3.136 & 2.493 & & 0.212 \\
Age & 0.040 & 0.020 & 0.286 & $0.05 \mathrm{I}$ \\
RHGR & -0.702 & 0.162 & -0.405 & $0.001 *$ \\
A-BEST & 0.038 & 0.059 & 0.093 & 0.524 \\
\hline
\end{tabular}

Notes: ${ }^{*} \mathrm{p}<0.05 ; B=$ unstandardized regression coefficient; $\mathrm{SE}_{B}=$ standard error of the coefficient; $\beta=$ standardized coefficient; Model 2 was adjusted for diabetes. Abbreviation: RHGS, relative handgrip strength.

equation $(p=0.001)$. An increase in RHGS of $1 \mathrm{~kg} / \mathrm{BMI}$ is associated with a decrease in TUG-test of $0.70 \mathrm{~s}$.

\section{Discussion}

The present study demonstrates that RHGS performs better in predicting impaired mobility evaluated by TUG-test in older women when compared with chronological age and physiological age (i.e. A-BEST). ${ }^{16}$ In addition, RHGS remained significantly associated with TUG-test, even after adjustment for diabetes.

According to Cruz-Jentoft et $\mathrm{al}^{27}$ muscle strength is an important predictor of poor patient outcome as increased functional limitation and when choosing tools for measurement of physical performance in clinical practice, handgrip strength might represent an important and simple tool to identify subjects with increased risk of impaired mobility. Recently, Silva et al ${ }^{18}$ demonstrated that older women with high RHGS presented a higher peak $\mathrm{O}_{2}$ consumption, a higher chronotropic index, and a better heart rate recovery in the first and second minutes. Furthermore, subjects with higher levels of handgrip strength are significantly more likely to have lower levels of systemic inflammatory markers, C-reactive protein and fibrinogen at follow-up. ${ }^{9}$ In addition, grip strength is inversely associated with risk mortality in females only. ${ }^{9}$

Independent of A-BEST or RHGS values, those with abnormal HRR after a stress test (one of the parameters included for the calculation of physiological age) are older, are more likely to have hypertension, diabetes and to smoke. $^{26}$ In addition, those with low RHGS are more likely to be obese, to have reduced physical function and less independence in daily living. ${ }^{28}$

As a weak handgrip strength is associated with lower physical performance, impaired heart rate recovery, low chronotropic index, and death even after adjustments for age, sex, country income level, education level, employment status, tobacco, alcohol use, diabetes, heart failure, 
coronary artery disease, chronic obstructive, pulmonary disease, self-reported prior stroke, self-reported prior cancer, body mass index, and waist-to-hip ratio. ${ }^{5,14,18}$ It is reasonable to suggest that our findings provide evidence that RGHS is highly associated with the TUG-test, which has been utilized in predicting the risk of falls in community-dwelling older adults. ${ }^{10,11}$

The scale is more easily understood by both patients and clinicians, and is more easily administered than the A-BEST. For example, it might be more relevant to tell a 67-year-old patient who achieves a RHGS of $1.48 \mathrm{~m}^{2}$ (low RHGS) that an increase in one unit $(\mathrm{kg} / \mathrm{BMI})$ diminishes the TUG-test in $0.70 \mathrm{~s}$ and increases mobility, but such approach must be explored in subjects perception in future studies. ${ }^{13}$

Furthermore, handgrip strength is a powerful predictor of poor patient outcomes such as longer hospital stays, increased functional limitation, poor health-related quality of life and death. ${ }^{9,13,14,29}$ In addition, handgrip strength does not require highly trained personal, and is an inexpensive tool that is simple and portable. Lastly, handgrip strength represents a noninvasive measurement for a clinician when compared with the stress testing which most older persons are unable to satisfactorily complete. ${ }^{8,13-17}$

Our study has some limitations. First, it was conducted in a small sample size of older women. Second, this correlational analysis on a cross section of older women cannot be utilized to uncover any direct causes of low handgrip strength and decreased mobility in this population. Third, we only included diabetes as the covariate variable; cause is an independent risk factor for reduced mobility and falls. ${ }^{12}$ However, other important risk factors as; dementia, previous falls, cardiotonic glycoside, neuroleptics and antidepressants were not controlled in our study. ${ }^{12}$ Thus, the hypothesis that RHGS is the main predictor of mobility in older women assessed by TUG-test needs to be confirmed in prospective studies.

A lot of independent risk factors are involved in reduced mobility and falls, and estimating chronological and physiological age is necessary for diagnoses and to determine whether an older subject tends to have increased risk for reduced mobility. However, the current use of 13 allostatic load markers or more to estimate the physiological age might not be always effective and the use of handgrip strength that outperforms traditional risk factors can help gauge as a new and important test to improve the health span of the older subjects. ${ }^{14}$ Because of this, we recommend that RGHS be utilized by clinicians as a convenient tool to predict impaired mobility and fall risk in older women and to include the low handgrip strength as a new allostatic load or a new index of physiological dysregulation that decreases up through $60 \mathrm{~s}^{30}$

\section{Conclusion}

In summary, RHGS better predicts impaired mobility evaluated by TUG-test in older women when compared with chronological age and physiological age.

\section{Acknowledgments}

The last author (DCN) would like to thank his family and in particular his mother Rita Cunha and his son Nicolas Cunha. Furthermore, we appreciate the assistance of Jeeser Alves de Almeida, PhD., during A-BEST interpretation.

\section{Author Contributions}

All authors contributed to article preparation, took part in drafting the article and reviewing critically for important intellectual content, gave final approval of the version to be published, and agree to be accountable for all aspects of the work.

\section{Disclosure}

The authors report no conflicts of interest in this work.

\section{References}

1. Chodzko-Zajko WJ, Proctor DN, et al.; American College of Sports Medicine Position Stand. Exercise and physical activity for older adults. Med Sci Sports Exerc. 2009;41(7):1510-1530. doi:10.1249/ MSS.0b013e3181a0c95c

2. Simões C. Relações Entre as Alterações Históricas Na Dinâmica Demográfica Brasileira E Os Impactos Decorrentes Do Processo De Envelhecimento Da População. Rio de Janeiro: IBGE; 2016:119.

3. McPhee JS, French DP, Jackson D, Nazroo J, Pendleton N, Degens H. Physical activity in older age: perspectives for healthy ageing and frailty. Biogerontology. 2016;17(3):567-580. doi:10.1007/s10522-016-9641-0

4. Levine ME. Modeling the rate of senescence: can estimated biological age predict mortality more accurately than chronological age? J Gerontol A Biol Sci Med Sci. 2013;68(6):667-674. doi:10.1093/ gerona/gls233

5. Kobayashi K, Imagama S, Ando K, et al. Weakness of grip strength reflects future locomotive syndrome and progression of locomotive risk stage: a 10-year longitudinal cohort study. Mod Rheumatol. 2019;24:1-7. doi:10.1080/14397595.2019.1626068

6. Blokh D, Stambler I. The use of information theory for the evaluation of biomarkers of aging and physiological age. Mech Ageing Dev. 2017;163:23-29. doi:10.1016/j.mad.2017.01.003

7. Brown PJ, Wall MM, Chen C, et al. Biological age, not chronological age, is associated with late-life depression. J Gerontol A Biol Sci Med Sci. 2018;73(10):1370-1376. doi:10.1093/gerona/glx162

8. Chainani V, Shaharyar S, Dave K, et al. Objective measures of the frailty syndrome (hand grip strength and gait speed) and cardiovascular mortality: a systematic review. Int $J$ Cardiol. 2016;215:487-493. doi:10.1016/j.ijcard.2016.04.068g 
9. Smith L, Yang L, Hamer M. Handgrip strength, inflammatory markers, and mortality. Scand J Med Sci Sports. 2019;29(8):1190-1196. doi:10.1111/sms.13433

10. Shumway-Cook A, Brauer S, Woollacott M. Predicting the probability for falls in community-dwelling older adults using the timed up \& go test. Phys Ther. 2000;80(9):896-903. doi:10.1093/ptj/80.9.896

11. Bischoff HA, Stahelin HB, Monsch AU, et al. Identifying a cut-off point for normal mobility: a comparison of the timed 'up and go' test in community-dwelling and institutionalised elderly women. Age Ageing. 2003;32(3):315-320. doi:10.1093/ageing/32.3.315

12. Bueno-Cavanillas A, Padilla-Ruiz F, Jimenez-Moleon JJ, PeinadoAlonso CA, Galvez-Vargas R. Risk factors in falls among the elderly according to extrinsic and intrinsic precipitating causes. Eur J Epidemiol. 2000;16(9):849-859. doi:10.1023/A:1007636531965

13. Lee WJ, Peng LN, Chiou ST, Chen LK. Relative handgrip strength is a simple indicator of cardiometabolic risk among middle-aged and older people: a nationwide population-based study in Taiwan. PLoS One. 2016;11(8):e0160876. doi:10.1371/journal.pone.0160876

14. Leong DP, Teo KK, Rangarajan S, et al. Prognostic value of grip strength: findings from the Prospective Urban Rural Epidemiology (PURE) study. Lancet. 2015;386(9990):266-273. doi:10.1016/S01406736(14)62000-6

15. Rijk JM, Roos PR, Deckx L, van den Akker M, Buntinx F. Prognostic value of handgrip strength in people aged 60 years and older: a systematic review and meta-analysis. Geriatr Gerontol Int. 2016;16(1):5-20. doi:10.1111/ggi.12508

16. Harb SC, Cremer PC, Wu Y, et al. Estimated age based on exercise stress testing performance outperforms chronological age in predicting mortality. Eur J Prev Cardiol. 2019:2047487319826400. doi:10. 1177/2047487319826400

17. Gill TM, DiPietro L, Krumholz HM. Role of exercise stress testing and safety monitoring for older persons starting an exercise program. JAMA. 2000;284(3):342-349. doi:10.1001/jama.284.3.342

18. Silva CR, Saraiva B, Nascimeno DD, et al. Relative handgrip strength as a simple tool to evaluate impaired heart rate recovery and a low chronotropic index in obese older women. Int J Exerc Sci. 2018;11(2):844.

19. Alonso A, Beunza JJ, Delgado-Rodriguez M, Martinez-Gonzalez MA. Validation of self reported diagnosis of hypertension in a cohort of university graduates in Spain. BMC Public Health. 2005;5:94. doi:10.1186/1471-2458-5-94
20. Lima-Costa MF, Peixoto SV, Firmo JO. [Validity of self-reported hypertension and its determinants (the Bambui study)]. Rev Saude Publica. 2004;38(5):637-642. doi:10.1590/S0034-89102004000500004

21. Nishime EO, Cole CR, Blackstone EH, Pashkow FJ, Lauer MS. Heart rate recovery and treadmill exercise score as predictors of mortality in patients referred for exercise ECG. JAMA. 2000;284 (11):1392-1398. doi:10.1001/jama.284.11.1392

22. Choquette S, Bouchard DR, Doyon CY, Senechal M, Brochu M, Dionne IJ. Relative strength as a determinant of mobility in elders 67-84 years of age. A nuage study: nutrition as a determinant of successful aging. J Nutr Health Aging. 2010;14(3):190-195. doi:10.1007/s12603-010-0047-4

23. Straight C, Brady A, Schmidt M, Evans E. Comparison of laboratory-and field-based estimates of muscle quality for predicting physical function in older women. J Aging Res Clin Pract. 2013;2 (3):276-279.

24. Vieira DCL, Madrid B, FdO P, et al. Ratings of perceived exertion in an incremental test in elderly women. Rev Bras Cineantropom Desempenho Hum. 2014;16(1):106-115. doi:10.5007/1980-0037.201 4v16n1p106

25. Silva C, Saraiva B, Nascimento DC, et al. Relationship between adiposity and heart rate recovery following an exercise stress test in obese older women. Rev Bras Cineantropom Desempenho Hum. 2017;19(5):554-564. doi:10.5007/1980-0037.2017v19n5p554

26. Cole CR, Foody JM, Blackstone EH, Lauer MS. Heart rate recovery after submaximal exercise testing as a predictor of mortality in a cardiovascularly healthy cohort. Ann Intern Med. 2000;132 (7):552-555. doi:10.7326/0003-4819-132-7-200004040-00041

27. Cruz-Jentoft AJ, Bahat G, Bauer J, et al. Sarcopenia: revised European consensus on definition and diagnosis. Age Ageing. 2019. doi:10.1093/ageing/afy169

28. Dong HJ, Marcusson J, Wressle E, Unosson M. Obese very old women have low relative handgrip strength, poor physical function, and difficulties in daily living. J Nutr Health Aging. 2015;19 (1):20-25. doi:10.1007/s12603-014-0512-6

29. Lawman HG, Troiano RP, Perna FM, Wang CY, Fryar CD, Ogden CL. Associations of relative handgrip strength and cardiovascular disease biomarkers in U.S. adults, 2011-2012. Am J Prev Med. 2016;50(6):677-683. doi:10.1016/j.amepre.2015.10.022

30. Crimmins EM, Johnston M, Hayward M, Seeman T. Age differences in allostatic load: an index of physiological dysregulation. Exp Gerontol. 2003;38(7):731-734. doi:10.1016/S0531-5565(03)00099-8

\section{Publish your work in this journal}

Open Access Journal of Sports Medicine is an international, peerreviewed, open access journal publishing original research, reports, reviews and commentaries on all areas of sports medicine. The manuscript management system is completely online and includes a very quick and fair peer-review system. Visit http://www.dovepress. com/testimonials.php to read real quotes from published authors. 